\title{
Wirtschaftlichkeitsprüfung, um die schwarzen von den weissen Schafen zu trennen
}

\author{
U. Vogt
}

Im vergangenen September hat unsere Geschäftsstelle santésuisse Basel im Rahmen des Wirtschaftlichkeitskontrollverfahrens eine Psychiaterin darauf aufmerksam gemacht, dass gemäss Rechnungsstellerstatistik der Krankenversicherer (Datenpool) die direkten sowie veranlassten Kosten ihrer Praxis stark vom Durchschnitt der Kosten ihrer Fachkolleginnen und -kollegen abweichen. Der Brief war knapp und schnörkellos gehalten und sorgte offenbar für Unmut, die Schweizerische Ärztezeitung (SÄZ) Nr. 48/2002 legt denn auch mehrfach Zeugnis davon ab. Ich will hier keine textkritische Analyse machen, erst recht nicht von den in der SÄZ veröffentlichten Beiträgen, die ja in einem Fall schon in der Basler Zeitung abgedruckt worden sind. santésuisse Basel hat diesen - ebenfalls in der Basler Zeitung - in angemessener Form beantwortet. Vielmehr soll es hier darum gehen, Sinn und Zweck der Wirtschaftlichkeitsprüfung zu beleuchten und damit auch die Qualität des Datenpools von santésuisse zu unterstreichen.

\section{Das Gesetz verpflichtet Kranken- versicherer zur Kontrolle}

Art. 56 Abs. 1 des Krankenversicherungsgesetzes sagt es deutlich: «Der Leistungserbringer muss sich in seinen Leistungen auf das Mass beschränken, das im Interesse der Versicherten liegt und für den Behandlungszweck erforderlich ist.» Gemäss dieser Bestimmung und der Rechtsprechung des Eidgenössischen Versicherungsgerichts (EVG) haben die Krankenversicherer nicht nur das Recht, sondern sogar die Pflicht, die Wirtschaftlichkeit der Leistungserbringer zu prüfen und allenfalls Massnahmen gegen eine unwirtschaftliche Praxisführung $\mathrm{zu}$ ergreifen. Was das genau bedeutet, darüber werden die Leistungserbringer bei einer Wirtschaftlichkeitskontrolle informiert. Das Verfahren wird im Prinzip in allen Kantonen gleich durchgeführt, kann sich allenfalls im Detail unterscheiden und erfolgt in der Regel mehrstufig. Eine interne Arbeitsgruppe von santésuisse arbeitet daran, das Verfahren gesamtschweizerisch zu optimieren. Betrachten wir hier nun das Beispiel Basel, des- sen Verfahren in einem Merkblatt zusammengefasst ist, welches im Sinne der Transparenz jeweils abgegeben wird:

Ausgangspunkt der Wirtschaftlichkeitskontrolle ist die Rechnungsstellerstatistik von santésuisse (Datenpool). Aus dieser werden die Kosten eines Arztes, einer Ärztin erhoben. Es wird dabei differenziert nach Ausbildung und Spezialität der betroffenen Arztperson, nach örtlichen Verhältnissen, nach der Zusammensetzung der Patientinnen und Patienten (insbesondere Durchschnittsalter) und nach weiteren als bedeutsam taxierten Faktoren, soweit solche bekannt sind. Stutzig werden die santésuisse-Spezialisten dann, wenn die solcherart ermittelten Arztkosten (eigentlich Arztrechnungen) markant höher sind als der Durchschnitt der Vergleichsgruppe. Die Vergleichsgruppe besteht dabei aus Ärztinnen und Ärzten der gleichen Fachrichtung und mit ähnlich zusammengesetzter Menge von Patientinnen und Patienten. Das verwendete Filtersystem orientiert sich an den Vorgaben des Eidgenössischen Versicherungsgerichts, welches definiert, wann eine ärztliche Behandlung als unwirtschaftlich zu taxieren ist.

Gibt es Abweichungen zwischen Index und Arzt X bzw. Ärztin Y analysieren die Fachleute von santésuisse die Situation umfassend unter Berücksichtigung der bekannten Fakten. Besteht der Verdacht auf Unwirtschaftlichkeit, schreibt santésuisse an die betroffene Arztperson einen Brief und macht sie auf die Auffälligkeit aufmerksam. Gleichzeitig wird auch zur vermehrten Beachtung des Gebots der Wirtschaftlichkeit hingewiesen und werden allfällige Rückforderungen für die kommenden Rechnungsjahre vorbehalten.

\section{Konzises Verfahren}

Die nach der zweiten Mahnung eintreffende Stellungnahme der Arztperson wird auf ihre Argumentation hin überprüft und in die Gesamtbeurteilung der Wirtschaftlichkeit der Praxisführung für die kommenden Jahre miteinbezogen. Falls die Vermutung auf unwirtschaftliche Praxisführung weiterbesteht, muss santésuisse entscheiden, ob detailliertere Abklärungen durch- 
zuführen sind. Erscheint der Fall als schwer wiegend, können ausdrücklich Rückforderungen für das nächste Jahr angedroht werden. Je nach Bedarf findet ein Gespräch statt. Können die Differenzen zwischen Arztperson und santésuisse jedoch nicht beseitigt werden, und weist die Rechnungsstellerstatistik im Folgejahr wiederum Auffälligkeiten auf, die auf unwirtschaftliches Verhalten schliessen lassen, wird das Rückforderungsverfahren eingeleitet. santésuisse reicht dabei im Namen aller Versicherer eine Rückforderung bei der Paritätischen Vertrauenskommission (PVK) bzw. beim zuständigen Gericht ein. Die PVK gilt als erste Instanz, wo eine vertragliche Vereinbarung zwischen Leistungserbringer und Kostenträger existiert, die folgenden sind dann das Kantonale Schiedsgericht (Art. $89 \mathrm{KVG}$ ) und als letzte Instanz das Eidgenössische Versicherungsgericht (EVG). Im vertragslosen Zustand gelangt man direkt an das Kantonale Schiedsgericht.

\section{Weisse und schwarze Schafe}

In den beiden Basel oder z. B. auch im Kanton Bern hat santésuisse die Erfahrung gemacht, dass rund 90 Prozent aller Ärztinnen und Ärzte ihre Arbeit korrekt machen und die meisten, die auf einen erhöhten Index aufmerksam gemacht werden müssen, diesen nicht bewusst generieren. Dieses Sichtbarmachen von vergleichsweise $\mathrm{zu}$ hohen Arztkosten hat denn auch häufig präven- tive Wirkung, damit der Arzt oder die Ärztin überhaupt auf das Problem aufmerksam wird. Diese Präventivwirkung ist denn auch fast wichtiger als das Geld, welches - oft in aussergerichtlichen Vergleichen geschlossen - an die Krankenversicherer zurückfliesst. Im Kanton Bern beispielsweise sind zwischen Juli 2001 und Mai 2002 rund 700 000-800 000 Franken an die Krankenversicherer zurückbezahlt worden.

Die schwarzen Schafe hingegen, die hängenbleiben, sind oft tatsächlich rabenschwarz, wie die Kassensturz-Sendung vom 10. Dezember 2002 gezeigt hat. Zwei krasse Beispiele seien hier erwähnt: Ein Allgemeinpraktiker wies im Jahr einen Umsatz von einer Million Franken aus, seine Medikamentenkosten und seine Arztkosten waren dabei doppelt so hoch wie jene seiner Kollegen im Durchschnitt. Ein zweiter Fall betraf ebenfalls einen Hausarzt: Dieser wies in einem Jahr neben stark überhöhten Medikamentenkosten rund 11000 Patientenkontakte aus - man stelle sich ein solches Arbeitspensum vor! santésuisse ist überzeugt davon, dass solche Ausreisser dem Image einer ganzen Branche äusserst abträglich sind. Dies erst recht in einer Zeit, in welcher ein Grossteil der Bevölkerung unter den seit Jahren ungebrochen steigenden Gesundheitskosten und entsprechenden Prämien leidet. Nicht umsonst waren die «schwarzen Schafe» auch in der Wintersession und im Zusammenhang mit der 2. KVG-Revision, über die im Nationalrat debattiert worden ist, ein stark beachtetes Thema. 